\title{
Dynamical Behavior of Two Dimensional Hénon Maps
}

\author{
M. S. Islam and M. S. Islam* \\ Department of Mathematics, University of Dhaka, Dhaka - 1000, Bangladesh
}

\begin{abstract}
In this article, we study the two dimensional non-linear dynamical behavior of Hénon maps. We investigate the parameter values for which fixed points and periodic points of period two exist and study the dimension of the maps. We also investigate the numerical results of the maps and use computer programming Mathematica for generating graphs and computations.
\end{abstract}

Keywords: Hénon attractor, Lorenz attractor, Strange attractor, Dimension, Fractal.

\section{Introduction}

Michel Hénon, an astronomer in Nice, France, curious about the degradation of celestial orbits, the orbits of Stars around the centers of their galaxies. He considered gravitational centers as a three dimensional object and carefully studied the orbits of the Stars. Hénon (Hénon, 1976) studied the map and continued plotting the points for a system with increased energy levels. He tried many ways to predict the upcoming points of his high-energy graph, he decided to abandon classical methods and used difference equations. He tried to understand the structure of the Lorenz attractor by a Poincaré map. The model of the polynomial map is given by

$(x, y) \propto\left(1+y-a x^{2}, b x\right)$ where are parameters.

Hénon found numerically an attractor with and $a=1.4$ and $b=0.3$, later called the Hénon attractor. The mathematical nature of this attractor is still not known, but Benedicks and Carleson (Benedicks and Carleson, 1991) proved the existence of attractor as the closure of a branch of the unstable manifold. They identified a set of parameters of positive Lebesgue measure for which the map admits a strange attractor.

Meteorologist Lorenz (Lorenz, 1963) published a numerical study of the ordinary differential equations

$$
\begin{aligned}
& x^{\prime}=\sigma(y-x) \\
& y^{\prime}=\rho x-y-x z \\
& z^{\prime}=-\beta z+x y
\end{aligned}
$$

where $\sigma, \beta, \rho>0$ are parameters, obtained from the Oberbeck-Boussinesq equations for fluid convection in a two-dimensional layer heated from below and cooled from above. Lorenz used the parameters $\sigma=10, \beta=8 / 3$ and $\rho=$ 28 and found a strange attractor, later called the Lorenz attractor, where the solutions seemed to be attracted to a branched manifold and orbits were sensible to initial conditions.

The Hénon map is the most studied examples of dynamical systems that exhibit chaotic behavior. The map has been the source of many numerical experiments and theoretical works. An equivalent formulation of the Hénon map is given by $(x, y) \mapsto\left(a+b y-x^{2}, x\right)$ where $a, b$ are parameters.

In the article (Niger et. al., 2008), investigated the parameter values for which fixed points and periodic points exists. We also studied the saddle node and period doubling bifurcation of the maps.

In this article, we study the numerical behavior and dimension of the maps, especially, we show the box-counting, Lyapunov and correlation dimensions. These dimensions are purely geometric, making no mention of the measure of the attractor, that is, the number of times the dynamics visits different regions of the phase space. We show that the map is a fractal and the dimension of the map lies between 1 and 2 and the map is a chaotic system.

\section{Materials and Methods}

\section{Definition 1}

Let $f$ be a smooth map. The Lyapunov number $L\left(x_{1}\right)$ of the orbit $\left\{x_{1}, x_{2}, x_{3}, \ldots\right\}$ is defined as

$L\left(x_{1}\right)=\lim _{n \rightarrow \infty}\left(\left|f^{\prime}\left(x_{1}\right)\right| \ldots \ldots\left|f^{\prime}\left(x_{n}\right)\right|\right)^{\frac{1}{n}}$, if this limit exists. 


\section{Definition 2}

The Lyapunov exponent is defined as $h\left(x_{1}\right)=\operatorname{In} L\left(x_{1}\right)$

\section{Definition 3}

Let $f$ be a map of $\mathfrak{R}^{n}, n \geq 1$ and let $\left\{x_{1}, x_{2}, x_{3}, \ldots\right\}$ be a bounded orbit of $f$. Then the orbit is chaotic if (i) it is not asymptotically periodic, (ii) no Lyapunov number is exactly one, and (iii) $\mathrm{L}\left(x_{1}\right)>1$.

\section{Definition 4}

Let $f$ be a map of $\mathfrak{R}^{n}$ Consider an orbit with Lyapunov exponents $h_{1} \geq h_{2} \geq \ldots \geq h_{n}$, and let $p$ denote the largest integer such that $\sum_{i=1}^{p} h_{i} \geq 0$. Then the Lyapunov dimension $D_{L}$ of the orbit is given by

$$
D_{L}= \begin{cases}0, & \text { if no such } p \text { exists } \\ p+\frac{1}{\left|h_{p+1}\right|}, & \text { if } p<n \\ n \quad, \text { if } p=n .\end{cases}
$$

\section{Definition 5}

A set $S$ exhibits sensitive dependence if there exists $r>0$ such that for all $\varepsilon>0$ and $x \in S$ there exists $y \in S$ such that $|x-y|<\varepsilon$ and $\left|x_{n}-y_{n}\right|>r$ for some $n$.

\section{Definition 6}

Let $S$ be a bounded set in $\mathfrak{R}^{n}$. The dimension of $S$ is a grid of $n$ - dimensional boxes of side length $\varepsilon$ over $S$. Let $N(\varepsilon)$ be equal to the number of boxes of the grid that intersect $S$. Then the scaling law for the dimension $d$ gives

$$
d=\frac{\ln N(\varepsilon)-\ln C}{\ln (1 / \varepsilon)}
$$

where $C$ is a constant for all small $\varepsilon$, the contribution of the second term in the numerator of this formula will be negligible for small $\varepsilon$. A bounded set $S$ in $\mathfrak{R}^{n}$ has box counting dimension and

$$
\text { box } \operatorname{dim}(S)=\lim _{\varepsilon \rightarrow 0} \frac{\ln N(\varepsilon)}{\ln (1 / \varepsilon)}, \text { if the limit exists. }
$$

\section{Two Dimensional Hénon Maps}

Consider the two-dimensional quadratic family of Hénon map $H: \mathfrak{R}^{2} \rightarrow \mathfrak{R}^{2}$ defined by

$$
H_{a, b}(x, y)=\left(a+b y-x^{2}, x\right) \text {. }
$$

This equation was first investigated numerically in 1977 by the astronomer Hénon, who observed that they have attractors with very complicated dynamics. There exists two fixed points of the Hénon map. The points are

$$
x=\frac{(b-1) \pm \sqrt{(1-b)^{2}+4 a}}{2}
$$

Now, $\quad D H_{a, b}(x, y)=\left|\begin{array}{cc}-2 x & b \\ 1 & 0\end{array}\right|=b$ and characteristic equation is $\lambda^{2}+2 x \lambda-b=0$ then $\lambda=-x \pm \sqrt{x^{2}+b}$.

When, $|\lambda|>1 \Rightarrow a<\frac{-(1-b)^{2}}{4}$ or $\frac{3(b-1)^{2}}{4}<a$ and $|\lambda|<1 \Rightarrow a<\frac{3}{4}(b-1)^{2}$ or $a>\frac{-1}{4}(1-b)^{2}$. If $a<\frac{3}{4}(b-1)^{2}$ or $a>\frac{-1}{4}(1-b)^{2}$, then the fixed points are sink.

If $a<\frac{-(1-b)^{2}}{4}$ or $\frac{3(b-1)^{2}}{4}<a$, then the fixed points are source.

\section{For periodic points of period two:}

We have $H_{a b b}{ }^{2}(x, y)=(x, y)$.

So, $x_{1}=\frac{(1-b)+\sqrt{4 a-3(1-b)^{2}}}{2}$ then,

$y_{1}=\frac{(1-b)-\sqrt{4 a-3(1-b)^{2}}}{2}$

and $x_{2}=\frac{(1-b)-\sqrt{4 a-3(1-b)^{2}}}{2}$

then, $y_{2}=\frac{(1-b)+\sqrt{4 a-3(1-b)^{2}}}{2}$.

Now,

$$
\begin{array}{r}
D H_{a, b}^{2}\left(x_{1}, y_{1}\right)=D H_{a, b}\left(x_{2}, y_{2}\right) D H_{a, b}\left(x_{1}, y_{1}\right) \\
=\left|\begin{array}{cc}
4 x_{1} x_{2}+b & -2 b x_{2} \\
-2 x_{1} & 0
\end{array}\right|
\end{array}
$$

For eigenvalues,

$$
\lambda=\frac{\left(4 x_{1} x_{2}+b\right) \pm \sqrt{16 x_{1}{ }^{2} x_{2}{ }^{2}+24 b x_{1} x_{2}+b^{2}}}{2}
$$


If $|\lambda|<1$, then $a>\frac{5-11 b+12 b^{2}-4 b^{3}}{4(1-b)}$ or

$a>\frac{3-3 b-4 b^{2}+4 b^{3}}{4(1+b)}$

and $|\lambda|>1$, then $a<\frac{3-3 b-4 b^{2}+4 b^{3}}{4(1+b)}$ or

$a<\frac{5-11 b+12 b^{2}-4 b^{3}}{4(1-b)}$.

If $a>\frac{5-11 b+12 b^{2}-4 b^{3}}{4(1-b)}$ or $a>\frac{3-3 b-4 b^{2}+4 b^{3}}{4(1+b)}$,

then the periodic points of period two is sink.

If $a<\frac{3-3 b-4 b^{2}+4 b^{3}}{4(1+b)}$ or $a<\frac{5-11 b+12 b^{2}-4 b^{3}}{4(1-b)}$ then the periodic points of period two are source.

Theorem 1:

For $|b|<1$, such that $a_{0}(b)=-\frac{1}{4}(b+1)^{2}$

a) if $a<a_{0}(b), H_{a, b}$ has no fixed point,

b) if $a=a_{0}(b), H_{a, b}$ has exactly one fixed point,

c) if $a>a_{0}(b), H_{a, b}$ has exactly two fixed points.

Proof: The proof of the theorem is given in article (Niger et. al., 2008).

Proposition 1: Le $X=\frac{x}{a}, Y=-\frac{b y}{a}$. Then $H_{a, b}(x, y)$ is topologically conjugate to $H_{a, b}^{*}(X, Y)=\left(\begin{array}{c}1+Y-a X^{2} \\ b X\end{array}\right)$, where $a$ and $b$ are both non-zero.

Proof: The proof of the proposition can be found in (Devaney, 1992).

\section{Numerical Behavior of the Hénon Maps}

Mathematica has been employed for numerical computation. At first take initial value $x_{0}=0, y_{0}=0$ and we get the points $t_{0}=\{0,0\} ; t_{1}=\{1,0\}$; $t_{2}=\{-0.4,0.3\} ; \quad t_{3}=\left\{1.07,-0.12 ; t_{4}=\{-0.74,0.32\}\right.$. Then we take the initial value $x_{0}=0.1, y_{0}=0.1$ and we get the points $s_{0}=\{0.1,0.1\} ; s_{1}=\{1.09,0.03\}$; $s_{2}=\left\{-0.62,0.33 ; s_{3}=\{0.79,-0.19\} ; s_{4}=\{-0.5,0.24\}\right.$.

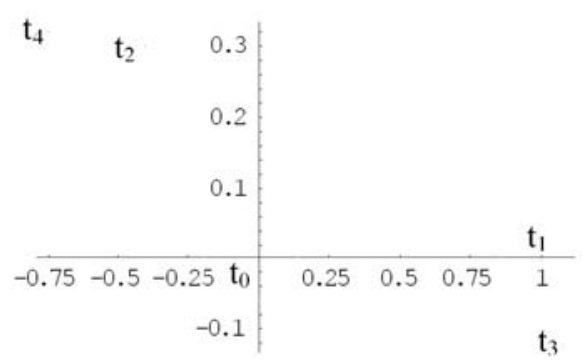

Fig. 1: Initial value $x_{0}=0, y_{0}=0$

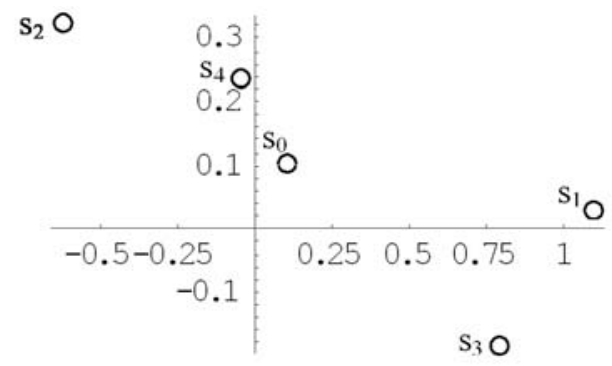

Fig. 3: Initial value $x_{0}=0.63, y_{0}=0.18$.

Again we take another experiment to get initial value $x_{0}=0.63, y_{0}=0.18$ and get another set of points $p_{0}=\{0.63,0.18\} ; p_{1}=\{.62434,0.189\} ;$

$p_{2}=\{0.643279,0.187302\}$;

$p_{3}=\{0.60797,0.192391\}$;

$p_{4}=\{0.675505,0.182391\}$.

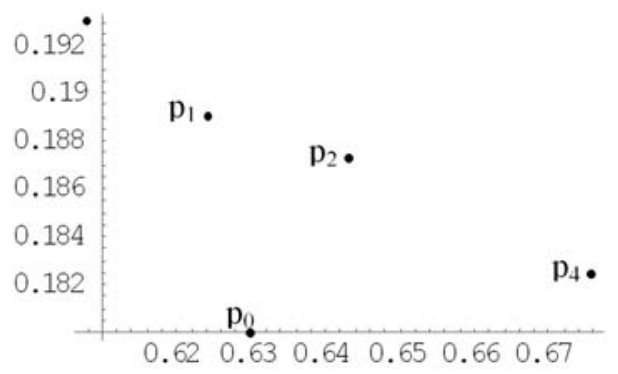

Fig. 3: Initial value $x_{0}=0.63, y_{0}=0.18$.

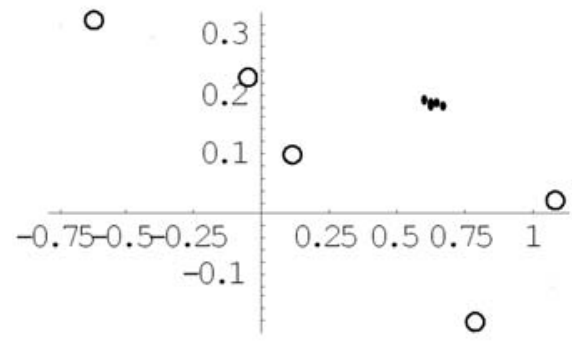

Fig 4: Combining Fig. 1, 2 and 3. 
From Fig. 4, it is observed that different sets of initial values give rise to various numbers of periodic points of period two. The numerical result of Fig. 5 shows the result of plotting 10000 successive points, obtained by iteration of the map starting from the arbitrarily chosen initial point $x_{0}=0, y_{0}=0$ the vertical scale is enlarged to give a better picture.

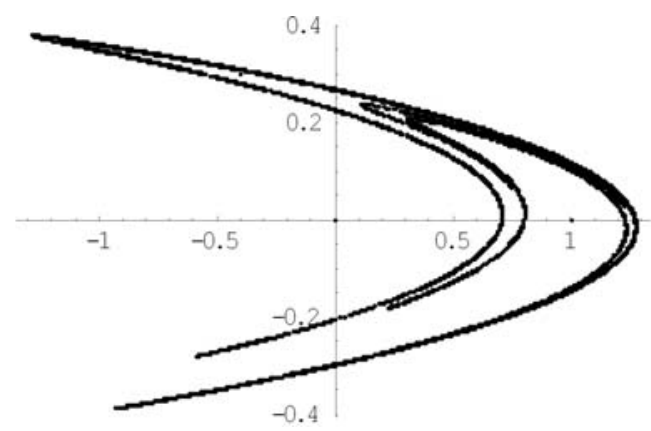

Fig. 5: 10000 successive points starting with $x_{0}=0, y_{0}=0$.

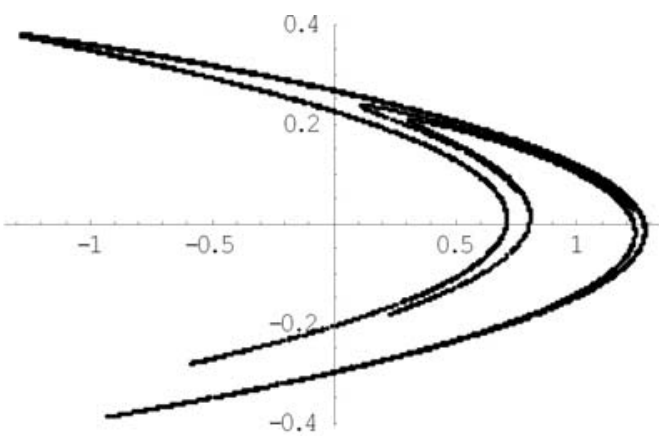

Fig. 6: 10000 successive points $x_{0}=0.63135448, y_{0}=0.18940634$.

Fig. 6 shows the result of 10000 iterations of $H$ aga starting from a different point

$x_{0}=0.63135448, y_{0}=0.18940634$.

The two figures are almost identical. This suggests stron€ that both figures are essentially the attractor it self. The init point at $x_{0}=0, y_{0}=0$ and the first iterate $x_{1}=1, y_{1}=0$ are clearly visible; the second iterate is $\mathrm{s}$ visible at $x_{2}=-0.4, y_{2}=0.3$; the third iterate can harc be distinguished at $x_{3}=1.706, y_{3}=-0.12$; and the fous iterate at $x_{4}=-0.7408864, y_{4}=0.3228$ is already $\mathrm{k}$ inside the attractor at the resolution of Fig. 5. The followi points then wander over the attractor in an apparently erra manner. Hénon map has two invariant points, given by

$$
x=(2 a)^{-1}\left[-(1-b) \pm \sqrt{(1-b)^{2}+4 a}\right], y=b x .
$$

These points are real for

$$
a>a_{0}=(1-b)^{2} / 4 .
$$

In this case, one of the points is always linearly unstable, while the other is unstable for

$$
a>a_{1}=3(1-b)^{2} / 4 \text {. }
$$

One of the two unstable invariant points has the coordinates $x_{0}=0.63135448 \ldots, y_{0}=0.18940634$.

The point appears to belong to the attractor. The two eigenvalues $\lambda_{1}, \lambda_{2}$ and the slopes $p_{1}, p_{2}$ of the corresponding eigenvectors are

$$
\begin{aligned}
& \lambda_{1}=0.15594632 \ldots, p_{1}=1.92373886 \ldots, \\
& \lambda_{2}=-1.92373886 \ldots, p_{2}=-0.15594632 \ldots
\end{aligned}
$$

The instability is due to $\lambda_{2}$. The corresponding slope

$p_{2}$ appears to be tangent to the curves in Fig. 6 .

\section{The Dimension of Hénon map}

Dimension (Pesion, 1997) of Hénon map is computed by the method of box-counting dimension and a comparison with that of Lyapunov dimension and correlation dimension.

Box-counting dimension is one of several definitions of fractal (Falconer, 1997) dimension. All the dimension do not give the same number. Some are easier to compute than others.

\section{Box-counting dimension of Hénon map}

For a more complicated example such as the Hénon attractor of $f(x, y)=\left(1.4-x^{2}+0.3 y, x\right)$,

no exact formula can be found for the box counting dimension. We draw pictures and counting boxes, and use the results to form an estimate of the dimension.

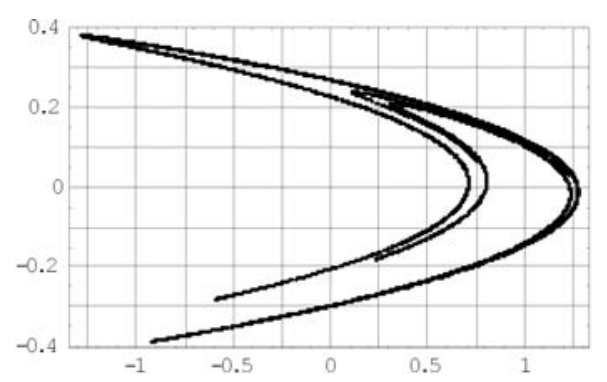

Fig.7: The Hénon attractor is shown in a grid of boxes with side-length $\varepsilon=1 / 4$. 


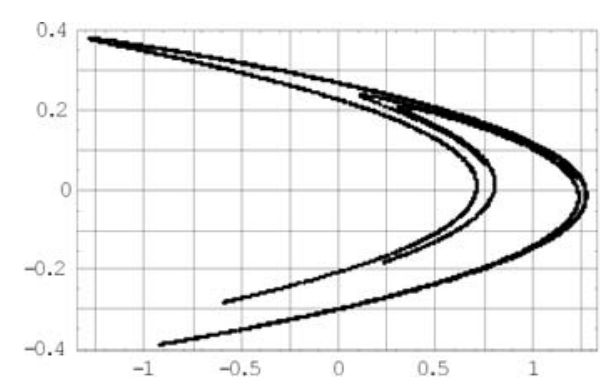

Fig. 8: The Hénon attractor is shown in a grid of boxes with side-length $\varepsilon=1 / 8$.

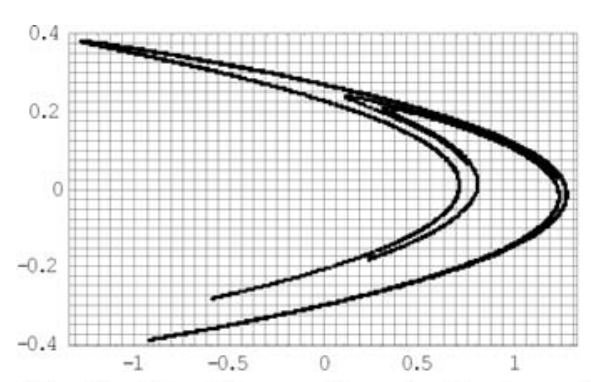

Fig. 11: The Hénon attractor is shown in a grid of boxes with side-length $\varepsilon=1 / 64$.

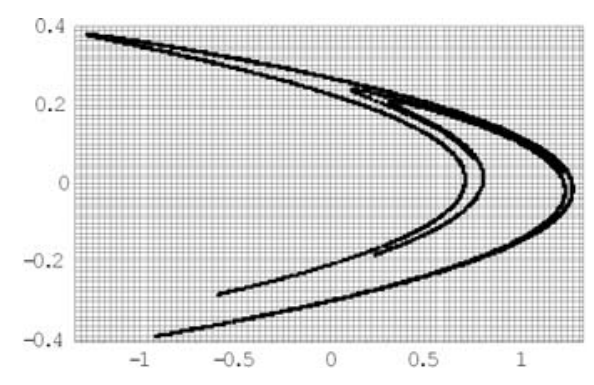

Fig. 12: The Hénon attractor is shown in a grid of boxes with side-length $\varepsilon=1 / 128$.

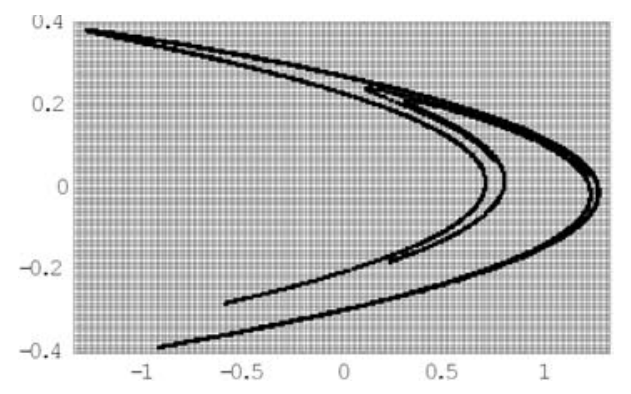

Fig. 11: The Hénon attractor is shown in a grid of boxes with side-length $\varepsilon=1 / 64$.

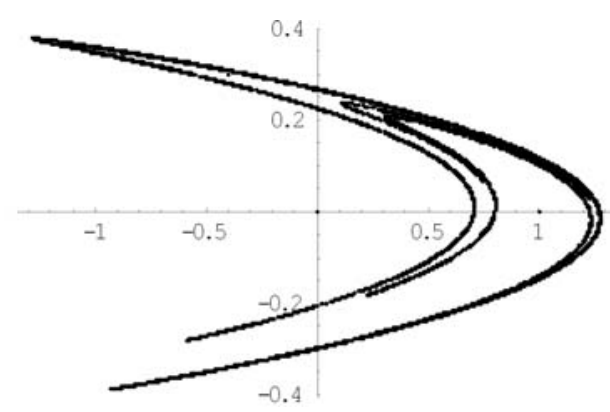

Fig. 12: The Hénon attractor is shown in a grid of boxes with side-length $\varepsilon=1 / 128$.

The Hénon attractor of $f(x, y)=\left(1.4-x^{2}+0.3 y, x\right)$ is shown beneath a grid of boxes with side length $\varepsilon=1 / 4$. Of the 256 boxes shown, 76 contain a piece of the attractor. Now we extend the grid of Fig. 7 to smaller boxes. The side length $\varepsilon$ of the boxes is $1 / 8$ and $1 / 16$ respectively in the two plots. A careful count reveals a total of 177 boxes hit by the attractor for $\varepsilon=1 / 8,433$ boxes hit by the attractor for $\varepsilon=1 / 16,1037$ for $\varepsilon=1 / 32,2437$ for $\varepsilon=1 / 64$ and 5763 for $\varepsilon=1 / 128$. In Fig. 13, we graph the quantity $\log _{2} N(\varepsilon)$ versus $\log _{2}(1 / \varepsilon)$ because its ratio is the same as $\frac{\ln N(\varepsilon)}{\ln (1 / \varepsilon)}$, which defines box-counting dimension in the limit as $\varepsilon \rightarrow 0$. We used box sizes $\varepsilon=2^{-2}$ through $\varepsilon=2^{-7}$, and take $\log _{2}$ of the box counts given above. The box counting dimension corresponds to the slope in the graph. Fig. 13 would be extended as far as possible to the right, in order to make the best approximation possible to the limit. The slope in the picture gives a value for the box-counting dimension approximately equal tol.27.

\section{Lyapunov dimension of Hénon map:}

In the case of a two dimensional $\operatorname{map} h_{1}>0>h_{2}$ and $\boldsymbol{h}_{1}+\boldsymbol{h}_{2}<0$ yields

$$
D_{L}=1+\frac{\boldsymbol{h}_{1}}{\left|\boldsymbol{h}_{2}\right|} .
$$

Consider the Hénon map with parameters $a=1.4$ and $b=0.3$. Typical initial conditions have Lyapunov exponents can be approximated by $\boldsymbol{h}_{1}=0.42$ and $\boldsymbol{h}_{2}=-1.62$. So, the Lyapunov dimension of the Hénon map is

$$
\begin{gathered}
D_{L}=1+\frac{\boldsymbol{h}_{1}}{\left|\boldsymbol{h}_{2}\right|} \\
\Rightarrow D_{L}=1+\frac{0.42}{|-1.62|} \Rightarrow D_{L}=1.259 .
\end{gathered}
$$




\section{Correlation dimension}

Correlation dimension is an altemate definition that is popular because of its simplicity and lenient computer storage requirements. It is different from box-counting dimension because it is defined for an orbit of a dynamical system. More generally, it can be defined for an invariant measure.

Let $S=\left\{x_{1}, x_{2}, \ldots \ldots ..\right\}$ be an orbit of the map $f$ on $\mathfrak{R}^{n}$. For each $r>0$, we define the correlation function $C(r)$ to be the proportion of pairs of orbit points within $r$ units of one another. Let $S_{N}$ denote the first $N$ points of the orbit $S$. Then

$C(r)=\lim _{n \rightarrow \infty} \frac{\left\{\text { pairs }\left(x_{1}, x_{2}\right): x_{1}, x_{2} \text { in } S_{N},\left|x_{1}-x_{2}\right|<r\right\}}{\left\{\text { pairs }\left(x_{1}, x_{2}\right): x_{1}, x_{2} \text { in } S_{N}\right\}}$

The correlation function $C(r)$ increases from 0 to 1 as $r$ increases from 0 to $\infty$. If $C(r)=r^{d}$ for small $r$, we say that the correlation dimension of the orbit $S$ is $d$. Then

$$
\text { cor } \operatorname{dim}=\lim _{r \rightarrow 0} \frac{\log C(r)}{\log (r)}, \text { if the limit exists. }
$$

Fig. 14 shows a measure of the correlation dimension of the orbit of the Hénon attractor. An orbit of length $N=1000$ generated, and $(1000)(999) / 2$ the possible pairs, the proportion that lie within $r$ was counted for $r=2^{-2}, \ldots \ldots \ldots, 2^{-8}$. According to the definition of correlation dimension, from the graph of $\log C(r)$ versus $\log r$ we try to estimate the slope as $r \rightarrow 0$. This estimate gives cor $\operatorname{dim}(S) \approx 1.23$ for the Hénon attractor, slightly less than the box-counting dimension estimate.

Dimension measurements in high-dimensional spaces, correlation dimension can be quite practical when compared with counting boxes. The number of $\varepsilon$-boxes in the unit "cube" of $\mathfrak{R}^{n}$ is $\varepsilon^{-n}$. If $\varepsilon=0.01$ and $n=10$, there are potentially $10^{20}$ boxes that need to be tracked.

The correlation dimension is characterized chaotic attractors was suggested by theoretical physicists (Grassberger and Procaccia, 1983).

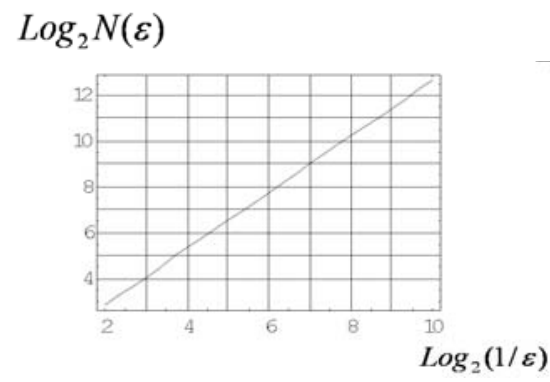

Fig. 13: Box counting dimension of the Hénon map

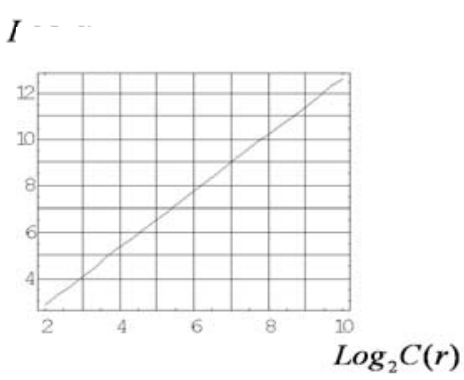

Fig. 14: Correlation dimension of Hénon map

\section{Conclusion}

We study the dynamical behavior of the Hénon maps. We show the numerical values of the parameters where the map has attractor and compare the different kinds of dimension of the maps. We show the dimension of the map:

\begin{tabular}{l|c|c}
\hline $\begin{array}{l}\text { Box-counting } \\
\text { dimension }\end{array}$ & $\begin{array}{c}\text { Lyapunov } \\
\text { dimension }\end{array}$ & $\begin{array}{c}\text { Correlation } \\
\text { dimension }\end{array}$ \\
\hline 1.27 & 1.259 & 1.23 \\
\hline
\end{tabular}

Hence, box-counting dimension is satisfactorily close to the Lyapunov dimension and correlation dimension. Although the box counting dimension is not exact method to find the dimension of the map. Since it has a common tool for the analysis of reconstruct attractors from experimental data.

\section{References}

Benedicks M. and L. Carleson (1991). The Dynamics of the Hénon Map, Ann. Math., 133: 73-169.

Devaney R. L. (1992). A First Course in Chaotic Dynamical Systems: Theory and Experiment, Perseus Books Publishing.

Falconer K. J. (1997). Techniques in Fractal Geometry, John Wiley \& Sons.

Grassberger P. and I. Procaccia (1983). Measuring the strangeness of strange attractors, Physica D, 9: 189-208.

Hénon M. (1976). A Two-dimensional Mapping with a Strange Attractor, Comm. Math. Phys., 69 - 77.

Lorenz E. N. (1963). Deterministic non Periodic Flow, $J$. Atomos. Sci., 130 - 141.

Niger A. M., Khan A. F. M. K. and Islam M. S. (2008). Periodic Points of a Two Dimensional Two Parameters Families of Hénon Maps, Bangladesh J. Sci. \& Res. 21(1 \& 2): 97-102.

Pesion Y. B. (1997). Dimension Theory in Dynamical Systems, Univ. Chicago Press, Chicago and London.

Received : February 15, 2010;

Accepted: December 19, 2010 